\title{
Germline cytotoxic lymphocytes defective mutations in Chinese patients with lymphoma
}

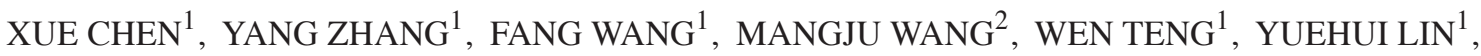 \\ XIANGPING HAN ${ }^{1}$, FANGYUAN JIN ${ }^{1}$, YUANLI XU ${ }^{1}$, PANXIANG CAO ${ }^{1}$, JIANCHENG FANG $^{1}$, \\ PING ZHU ${ }^{2}$, CHUNRONG TONG ${ }^{1}$ and HONGXING LIU ${ }^{1}$ \\ ${ }^{1}$ Department of Pathology and Laboratory Medicine Division, Hebei Yanda Lu Daopei Hospital, Sanhe, \\ Hebei 065201; ${ }^{2}$ Department of Hematology, Peking University First Hospital, Beijing 100034, P.R. China
}

Received February 25, 2016; Accepted July 7, 2017

DOI: $10.3892 / 01.2017 .6898$

\begin{abstract}
Certain patients with lymphoma may harbor mutations in perforin 1 (PRF1), unc-13 homolog D (UNC13D), syntaxin 11 (STX11), STXBP2 (syntaxin binding protein 2) or SH2 domain containing 1A (SH2D1A), which causes functional defects of cytotoxic lymphocytes. Data regarding the association between genetic defects and the development of lymphoma in Chinese patients are limited to date. In the present study, 90 patients with lymphoma were analyzed for UNC13D, PRF1, STXBP2, STX11, SH2D1A and X-linked inhibitor of apoptosis. Mutations were observed in $24(26.67 \%)$ patients; 16 patients exhibited mutations in UNC13D, 7 exhibited PRF1 mutations, and 1 exhibited monoallelic mutation in STX11. UNC13D c.2588G $>$ A/p.G863D mutation was detected in 9 patients (10.00\%) and in 4/210 controls (1.90\%). This mutation was predicted to be pathogenic and it predominantly existed in the Chinese population. These findings suggest that impaired cytotoxic machinery may represent a predisposing factor for the development of lymphoma. Furthermore, these data describe a distinct mutation spectrum in Chinese patients with lymphoma, whereby UNC13D is the most frequently mutated gene. In addition, these findings suggest UNC13D c. $2588 \mathrm{G}>\mathrm{A}$ mutation is a founder mutation in Chinese patients.
\end{abstract}

\section{Introduction}

The perforin-dependent granule-mediated cytolysis of cytotoxic lymphocytes (CLs), including natural killer cells and cytotoxic $\mathrm{T}$ lymphocytes, is the key machinery in the clearance of viral, and intracellular bacterial infections, as well as

Correspondence to: Dr Hongxing Liu, Department of Pathology and Laboratory Medicine Division, Hebei Yanda Lu Daopei Hospital, 6 Sipulan Road, Sanhe, Hebei 065201, P.R. China E-mail: lhongxing@outlook.com

Key words: cytotoxic lymphocytes, founder mutation, gene mutation, lymphoma in the prevention of tumor development $(1,2)$. The proteins encoded by perforin 1 (PRF1), unc-13 homolog D (UNC13D), syntaxin 11 (STX11), and STXBP2 (syntaxin binding protein 2) serve an essential role in this pathway. Mutations in these genes lead to function defects of CLs and are causative of familial hemophagocytic lymphohistiocytosis type 2 (FHL2), FHL3, FHL4, and FHL5 (3-6). The clinical manifestation of $\mathrm{X}$-linked lymphoproliferative disease (XLP), which is caused by mutations in $\mathrm{SH} 2$ domain containing $1 \mathrm{~A}$ (SH2D1A) (7) or $\mathrm{X}$-linked inhibitor of apoptosis (XIAP) (8) genes, resembles hemophagocytic lymphohistiocytosis. Furthermore, XLP2 due to XIAP deficiency has been suggested to be classified as X-linked FHL (9).

A proportion of patients with lymphoma have been reported to harbor mutations in PRF1, UNC13D, STX11, STXBP2 or SH2D1A genes (10-14), indicating that genetic defective function of CLs may increase susceptibility to lymphomagenesis. The aim of the present study was to investigate the association between mutations in genes involved in the cytotoxic function of CLs and the development of lymphoma in Chinese patients.

\section{Patients and methods}

Cases and controls. In the present study, 68 and 34 patients with lymphoma were admitted to Hebei Yanda Lu Daopei Hospital (Sanhe, China) and Peking University First Hospital (Beijing, China), respectively, between August 2013 and August 2015; 12/102 were excluded due to poor DNA quality. A total of 90 (61 from Hebei Yanda Lu Daopei Hospital and 29 from Peking University First Hospital) unrelated patients with lymphoma (48 males and 42 females; age range, 3-60 years) were recruited in the present study; 39 were diagnosed with Hodgkin lymphoma and 51 were diagnosed with non-Hodgkin lymphoma according to the World Health Organization classification (15). Healthy donors of Han nationality $(n=210)$ at the Hebei Yanda Lu Daopei Hospital served as controls. The present study was approved by the Ethics Committees of Hebei Yanda Lu Daopei Hospital and Peking University First Hospital. Written informed consent was obtained from all patients and healthy donors or their parents in accordance with the 1964 Helsinki declaration, and its later amendments or comparable ethical standards. 
Amplification and sequence analysis. Genomic DNA was isolated from peripheral blood and bone marrow using the TIANamp Blood DNA kit (item no. DP318; Tiangen Biotech Co., Ltd., Beijing, China) or from nails using the TIANamp FFPE DNA kit (item no. DP331; Tiangen Biotech Co., Ltd.) according to the manufacturer's protocol. Referenced coding sequences of the PRF1 (NM_005041.4), UNC13D (NM_199242.2), STXBP2 (NM_003764.3), STX11 (NM_006949.2), SH2D1A (NM_002351.3), and XIAP (NM_001167.2) were obtained from the National Center for Biotechnology Information Consensus CDS database (https://www.ncbi.nlm.nih.gov/projects/CCDS/CcdsBrowse.cgi). Primers were designed to amplify the coding exons and the flanking intron sequences by polymerase chain reaction (PCR). The sequences of primers are presented in Table I. The PCR system comprised of $1 \mu 1$ genomic DNA (10 ng/ $\mu \mathrm{l}), 1 \mathrm{ml}$ forward primer $(20 \mathrm{pmol} / \mu \mathrm{l}), 1 \mathrm{ml}$ reverse primer $(20 \mathrm{pmol} / \mu \mathrm{l}), 10 \mu \mathrm{l}$ Phusion Flash High-Fidelity PCR Master mix (Thermo Fisher Scientific, Inc., Waltham, MA, USA), and $7 \mu \mathrm{l}$ distilled water in a total volume of $20 \mu \mathrm{l}$. Reaction conditions were $10 \mathrm{sec}$ at $98^{\circ} \mathrm{C}$ followed by 38 cycles of $10 \mathrm{sec}$ at $98^{\circ} \mathrm{C}, 10 \mathrm{sec}$ at $68^{\circ} \mathrm{C}, 15 \mathrm{sec}$ at $72^{\circ} \mathrm{C}$, and then $1 \mathrm{~min}$ at $72^{\circ} \mathrm{C}$. The amplified PCR products were purified with ExoSAP-IT (USB Co., Cleveland, OH, USA) and followed by cycle sequencing PCR using a BigDye Terminator Sequencing Kit version 3.1 (Thermo Fisher Scientific, Inc.). Fluorescent labeled products were separated using an ABI 3500xL Genetic Analyzer (Thermo Fisher Scientific, Inc.). Variations were analyzed using Variant Reporter software (version 1.1; Thermo Fisher Scientific, Inc.). Genetic polymorphism information from the Single Nucleotide Polymorphism database (dbSNP; http://www. ncbi.nlm.nih.gov/snp/), 1000 Genomes Project (http://www. ncbi.nlm.nih.gov/variation/tools/1000genomes/) and the Exome Aggregation Consortium (ExAC; http://exac.broadinstitute. org/) were referenced to obtain the frequencies of variants in large populations. Variants with minor allele frequencies $>1 \%$ in the 1000 Genomes Project and/or ExAC were regarded as SNPs rather than mutations.

Confirmation of germline derivation of mutations. For patients determined to harbor mutations, the same mutation was detected in the DNA isolated from peripheral blood of their parents. In the absence of one or both parents, the detection of the same mutation in DNA extracted from nails of the patients could be of value. This was performed in order to confirm that the mutations were germline-derived.

In silico analysis. Two bioinformatics tools were used to predict whether an amino acid substitution was benign or deleterious: Sorting Intolerant From Tolerant (SIFT; http://sift.jcvi.org/) predicts whether an amino acid substitution affects protein function based on the degree of conservation of amino acid residues in multiple sequence alignments derived from closely associated sequences (16); and Polymorphism Phenotyping version 2.0 (PolyPhen-2; http://genetics.bwh.harvard.edu/pph/) predicts the possible impact of an amino acid substitution on the structure and function of a human protein using straightforward physical and comparative analyses (17). Iterative Threading ASSEmbly Refinement (I-TASSER; http://zhanglab.ccmb.med. umich.edu/I-TASSER/) was also used to predict and simulate the influence of the variants in protein tertiary structures.
Statistical analysis. Comparisons of mutant frequencies as well as genotype distributions between patients with lymphoma and controls were performed using the Chi-square test with SPSS software (version 20.0; IBM Corp., Armonk, $\mathrm{NY}$, USA). $\mathrm{P}<0.05$ was considered to indicate a statistically significant difference.

\section{Results}

Analysis of the gene mutations. A total of 18 different mutations were identified in 24 unrelated patients (26.67\%) (Fig. 1). A total of 16 patients $(17.78 \%$ ) carried mutations in UNC13D, including 12 with monoallelic mutations, 1 with homozygous mutation and 3 with compound heterozygous mutations. Seven patients $(7.78 \%)$ had PRF1 mutations, including 4 with monoallelic mutations, 1 with homozygous mutation and 2 with compound heterozygous mutations. One patient $(1.11 \%)$ was detected to carry STX11 monoallelic mutation (Table II). All mutations were confirmed to be germline-derived.

Sixty unrelated healthy donors were sequenced for these 6 genes with the same methods and 5 of them $(8.33 \%)$ were detected to harbor mutations. All 5 individuals were heterozygous for UNC13D mutations (c.680G>A/p.R227H; c.3134C $>$ T/p. T1045M; c.3229_3235del/p.Arg1077SerfsTer48; c.2553+5C>G; c.602A>G/p.H201R).

The Chi-square test revealed that the difference between mutant frequencies of patients with lymphoma and healthy donors was of statistical significance $(\mathrm{P}=0.005)$. Individuals carrying mutations of these genes were more likely to develop lymphoma compared with those without mutations [odds ratio (OR), 4.000; 95\% confidence interval (CI), 1.431-11.180].

Statistical analysis of UNC13D c.2588G $>$ A mutation. UNC13D c. $2588 \mathrm{G}>\mathrm{A} / \mathrm{p}$.G863D was the most frequent mutation identified in the current study, which was identified in 9 patients $(10.00 \%)$, including 1 homozygous and 8 heterozygous. This genetic variation was annotated as rs140184929 in dbSNP without frequency data. Data in the 1000 Genomes Project demonstrated that the c.2588A allele existed predominantly in the Chinese $(0.83 \%)$, and rarely in the Japanese $(0.48 \%)$ and Bengali $(0.58 \%)$ populations. Other populations did not carry this variant (Table III). Data in ExAC also demonstrated that the allelic frequency of c. $2588 \mathrm{~A}$ was increased in East Asian populations $(37 / 8,638$; $0.43 \%$ ) compared with that in South Asian populations $(5 / 16,504$; $0.03 \%$ ). Only one individual out of 32,962 Europeans was heterozygous for c. $2588 \mathrm{G}>\mathrm{A}$ variant. This variation was not observed among 14,554 individuals analyzed from other populations. Considering the high allele frequency of this mutation in the present patient cohort and the distinctly different allele frequencies among diverse populations, genotyping of the c.2588 allele was performed in 210 unrelated healthy donors of Chinese Han nationality (Table III). Heterozygous c. $2588 \mathrm{G}>\mathrm{A}$ was observed in 4 of them. Combined with data in the 1000 Genomes Project (a total of 301 Chinese), a control cohort of 511 individuals, 9 of whom harbored c.2588A allele in a heterozygous state was obtained. The Chi-square test revealed that the allele frequency of c.2588A in patients was significantly increased compared with that in the control group $(\mathrm{P}<0.001$; OR, 6.621; 95\% CI, 2.652-16.532), suggesting an association between the c.2588G $>\mathrm{A}$ mutation, and the risk of developing lymphoma. 
Table I. Primers used for amplification of the coding exons and the flanking intron sequences of perforin 1, unc-13 homolog D, syntaxin binding protein 2 , syntaxin 11, SH2 domain containing $1 \mathrm{~A}$ and $\mathrm{X}$-linked inhibitor of apoptosis.

\begin{tabular}{|c|c|}
\hline Name of the primer & Sequence $5^{\prime}$ to $3^{\prime}$ \\
\hline UNC13D-1FS & TGTAAAACGACGGCCAGTACTCGAGGAAGTGGGGTGAGA \\
\hline UNC13D-1RS & CAGGAAACAGCTATGACCGAGACCACAGTGCTCCCCAA \\
\hline UNC13D-2FS & TGTAAAACGACGGCCAGTCCTGTCCATCTGAGCCTGCTC \\
\hline UNC13D-2RS & CAGGAAACAGCTATGACCGGGACCCCACCCCATGCTCA \\
\hline UNC13D-3FS & TGTAAAACGACGGCCAGTGGTCAGGGAGCTTGAGGTAACC \\
\hline UNC13D-3RS & CAGGAAACAGCTATGACCAGACCCTGCTACCCAGGAAAG \\
\hline UNC13D-4FS & TGTAAAACGACGGCCAGTGCTCTGGGCTGTGGTCACTTAC \\
\hline UNC13D-4RS & CAGGAAACAGCTATGACCAGGCTCAGCTTTGTGAGGACAC \\
\hline UNC13D-5FS & TGTAAAACGACGGCCAGTCCTGGGGTCCACCTCCTGTC \\
\hline UNC13D-5RS & CAGGAAACAGCTATGACCGCTGGTGGCTCAGGGGTTC \\
\hline UNC13D-6FS & TGTAAAACGACGGCCAGTGGCAATTTCCTCСТCССТGTC \\
\hline UNC13D-6RS & CAGGAAACAGCTATGACCCAGTGGTGCCAGTCTGTCGAC \\
\hline UNC13D-7FS & TGTAAAACGACGGCCAGTGCAGGGTCCTGGTACAGATGTG \\
\hline UNC13D-7RS & CAGGAAACAGCTATGACCGCCATGGAGAAGAGGTGGATC \\
\hline UNC13D-8FS & TGTAAAACGACGGCCAGTGGTGTATGCCACTGGGTGACA \\
\hline UNC13D-8RS & CAGGAAACAGCTATGACCAGGTCCAGGCAGAACCCAAG \\
\hline UNC13D-9FS & TGTAAAACGACGGCCAGTCTGGTGATGGTAGCTGCTCTATGA \\
\hline UNC13D-9RS & CAGGAAACAGCTATGACCCAGCTGGGACAGAGATGCAGA \\
\hline UNC13D-10FS & TGTAAAACGACGGCCAGTCCAGGCAGCCAACATGGTAA \\
\hline UNC13D-10RS & CAGGAAACAGCTATGACCAGAGAACATGCTTTGCCTGGTC \\
\hline UNC13D-11FS & TGTAAAACGACGGCCAGTCTACAAACTGCTCTCACAGAACGG \\
\hline UNC13D-11RS & CAGGAAACAGCTATGACCGGCTGCTACACCCCTCAGAAC \\
\hline UNC13D-12FS & TGTAAAACGACGGCCAGTGAGCGTCTTTGCTTCCTCCTC \\
\hline UNC13D-12RS & CAGGAAACAGCTATGACCGCTCACTGTCAAGGGTAACATGTC \\
\hline UNC13D-13FS & TGTAAAACGACGGCCAGTTCCCATGACCCAАTACTTTCCA \\
\hline UNC13D-13RS & CAGGAAACAGCTATGACCGCACTGACCCCTCCTGGTAAC \\
\hline UNC13D-14FS & TGTAAAACGACGGCCAGTACTCATCCGGAAGTACTTCTGCA \\
\hline UNC13D-14RS & CAGGAAACAGCTATGACCCACATCCAGCTGCAAACTCTTG \\
\hline UNC13D-15FS & TGTAAAACGACGGCCAGTAGCTGGCTTTGCAGTCCAAA \\
\hline UNC13D-15RS & CAGGAAACAGCTATGACCTCAGACCGTTGCTGGTATCAAA \\
\hline UNC13D-16FS & TGTAAAACGACGGCCAGTGGAGAAGGGCCTGGATCTCA \\
\hline UNC13D-16RS & CAGGAAACAGCTATGACCCCTACAGGAAAGCCCTTGCA \\
\hline STXBP2-1FS & TGTAAAACGACGGCCAGTGACTCAACTTCCTGGGCCTG \\
\hline STXBP2-1RS & CAGGAAACAGCTATGACCGGAGCAGCTGAGGCCGGAACT \\
\hline STXBP2-2FS & TGTAAAACGACGGCCAGTTGGTGGGACCAGAGAACCAG \\
\hline STXBP2-2RS & CAGGAAACAGCTATGACCCACGCTCAGGTCCCATCTCA \\
\hline STXBP2-3FS & TGTAAAACGACGGCCAGTTGGTGGTCCCTAAGTGGGTTTC \\
\hline STXBP2-3RS & CAGGAAACAGCTATGACCGCATACACACACGCTCACTCATG \\
\hline STXBP2-4FS & TGTAAAACGACGGCCAGTCCATGTGGGTGCGACACTAGT \\
\hline STXBP2-4RS & CAGGAAACAGCTATGACCGCCCAGCCTCAGTGTCTGTTT \\
\hline STXBP2-5FS & TGTAAAACGACGGCCAGTCAACCCTGGTGCTTCTGTCC \\
\hline STXBP2-5RS & CAGGAAACAGCTATGACCGGAACCAGGTCAGTGGCAAG \\
\hline STXBP2-6FS & TGTAAAACGACGGCCAGTCTTGCCACTGACCTGGTTCC \\
\hline STXBP2-6RS & CAGGAAACAGCTATGACCGAACGCAGACAGAGCATGGG \\
\hline STXBP2-7FS & TGTAAAACGACGGCCAGTCCGCAGTACCAGAAGGAGCT \\
\hline STXBP2-7RS & CAGGAAACAGCTATGACCСССТССАССТСТССАCAAGC \\
\hline STXBP2-8FS & TGTAAAACGACGGCCAGTCCTTGAGAGACCTGGTGCTGAG \\
\hline STXBP2-8RS & CAGGAAACAGCTATGACCGTGGGAGACGCTGGCAAATG \\
\hline STXBP2-9FS & TGTAAAACGACGGCCAGTCCAGGTTTCCCACTCTTGCTC \\
\hline STXBP2-9RS & CAGGAAACAGCTATGACCGACCAGACCCGAAACACTGC \\
\hline STXBP2-10FS & TGTAAAACGACGGCCAGTTCTGTGACCAGCCTCCТTCC \\
\hline
\end{tabular}


Table I. Continued.

Name of the primer

Sequence $5^{\prime}$ to $3^{\prime}$

STXBP2-10RS

STXBP2-11FS

STXBP2-11RS

STXBP2-12FS

STXBP2-12RS

STX11-1FS

STX11-1RS

STX11-2FS

STX11-2RS

PRF1-1FS

PRF1-1RS

PRF1-2FS

PRF1-2RS

PRF1-3FS

PRF1-3RS

SH2D1A-1FS

SH2D1A-1RS

SH2D1A-2FS

SH2D1A-2RS

SH2D1A-3FS

SH2D1A-3RS

SH2D1A-4FS

SH2D1A-4RS

XIAP-1FS

XIAP-1RS

XIAP-2FS

XIAP-2RS

XIAP-3FS

XIAP-3RS

XIAP-4FS

XIAP-4RS

XIAP-5FS

XIAP-5RS

XIAP-6FS

XIAP-6RS

XIAP-7FSa

XIAP-7RSa

\begin{abstract}
CAGGAAACAGCTATGACCCCTCAGCAGAGCAGATCGGT
TGTAAAACGACGGCCAGTCAGAGGCAGGAGGTGGAGATG

CAGGAAACAGCTATGACCTGTCCCTGTCCCTCAGCAAA

TGTAAAACGACGGCCAGTAAGTGGGAGGTGCTCATTGG

CAGGAAACAGCTATGACCAAGTCCAAGTTCTTAACCTCCATGA

TGTAAAACGACGGCCAGTTTGCCCACACCGAGGAATAC

CAGGAAACAGCTATGACCCTCGCTCAGCTCCTTCATGG

TGTAAAACGACGGCCAGTGCGAGGTCATCCACTGCAAG

CAGGAAACAGCTATGACCCTTTGGTGCGTCCTTCCCAG

TGTAAAACGACGGCCAGTCCTTCCATGTGCCCTGATAA

CAGGAAACAGCTATGACCGCCAGGATTGCAGTTTCTTC

TGTAAAACGACGGCCAGTCCCTGGGTTCCAGTCCTAGT

CAGGAAACAGCTATGACCGCCCTGTCCGTCAGGTACT

TGTAAAACGACGGCCAGTCTGCACGTGCTGCTGGACA

CAGGAAACAGCTATGACCCTGGTCCTTTCCAAGCTCAC

TGTAAAACGACGGCCAGTGCTCGATCGAACCAAGCTAC

CAGGAAACAGCTATGACCGGATTGAGGCGAAAGTGTGT

TGTAAAACGACGGCCAGTTCTCACTGGAAACTGTGGTTGG

CAGGAAACAGCTATGACCGCTAAACAGGACTGGGACCAAA

TGTAAAACGACGGCCAGTACTTCTCTTAGCATCCCTAGCAC

CAGGAAACAGCTATGACCCTGGCTACATCTACTTTCTCACTGC

TGTAAAACGACGGCCAGTAGGCTCAGGCATAAACTGAC

CAGGAAACAGCTATGACCGCATTTGTAGCTCACCGAACTGT

TGTAAAACGACGGCCAGTAGAATGTTTCTTAGCGGTCGTGTAG

CAGGAAACAGCTATGACCGTTCCTCGGGTATATGGTGTCTGATAT

TGTAAAACGACGGCCAGTTCTGGGAAGCAGAGATCATTTTG

CAGGAAACAGCTATGACCCCTGGCATACTTGGGAAGCT

TGTAAAACGACGGCCAGTAGTGTGTATTTCTTCCTCAAAGGATAA

CAGGAAACAGCTATGACCCTCCCACTGCATGCTATCCAA

TGTAAAACGACGGCCAGTCAGTGGGATAGGGAATTGGGTA

CAGGAAACAGCTATGACCCACTGCCCAGCTAGCTCTCAT

TGTAAAACGACGGCCAGTGGTGGCCAAGGCATCAGTAA

CAGGAAACAGCTATGACCGCGCATCACAAGATCAGGAGT

TGTAAAACGACGGCCAGTACCCGCTCTGCTACAGAAAC

CAGGAAACAGCTATGACCCACATCTGGCCCTTTCTTGCTTT

TGTAAAACGACGGCCAGTCAGATGCCACGGGTGAGTCA

CAGGAAACAGCTATGACCATTGCCAACTAAAACACTGCCAT
\end{abstract}

The segment in bold font is a nonspecific tail named S1, which is added to the specific forward primers. The segment in italic font is a nonspecific tail named S2, which is added to the specific reverse primers. S1 and S2 are also used as sequencing primers.

In silico analysis of UNC13D c.2588G $>A$ mutation. The UNC13D c.2588G $>$ A/p.G863D mutation resulted in a substitution of the nonpolar and hydrophobic glycine (often involved in the formation of the turn structure) in the Munc13 homology domain 2 of protein UNC13D by the polar, and neutral aspartic acid (often involved in the formation of the coil structure). Multiple sequence alignment demonstrated that the amino acid at this position was highly conserved in available vertebrate species (Fig. 2A) and the alteration is predicted to be possibly damaging using PolyPhen-2 (Fig. 2B), and deleterious with SIFT in silico analysis. I-TASSER also demonstrated significant differences in the 3D structures of the wild-type and mutant-type proteins (Fig. 2).

\section{Discussion}

In 2005, Clementi et al (10) first reported that 8/29 (27.6\%) unrelated Italian patients with lymphoma carried PRF1 mutations and 5 of them carried PRF1 c.272C $>$ T/p.A91V heterozygous mutation. In 2014, Ciambotti et al (11) observed mutations in 23/84 (27.4\%) Italian patients with anaplastic large cell lymphoma following genotype analysis of PRF1, 


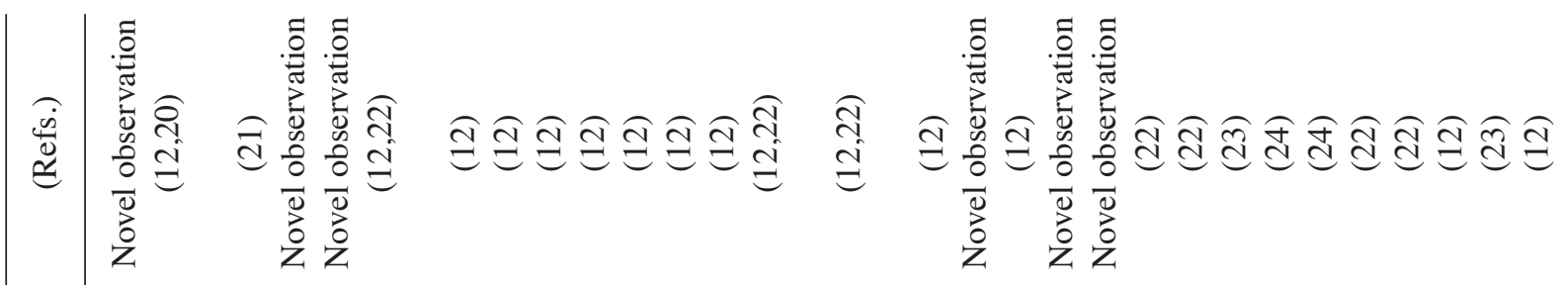

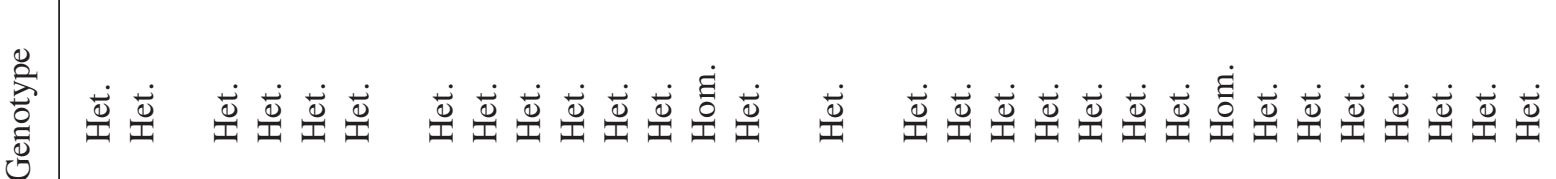

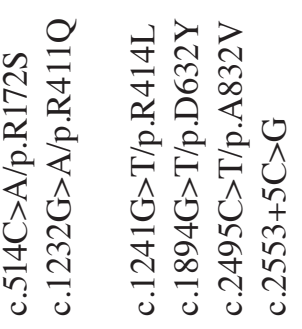

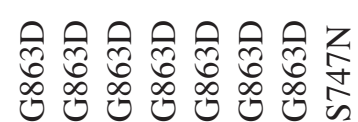

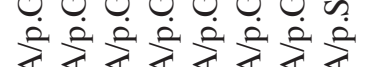

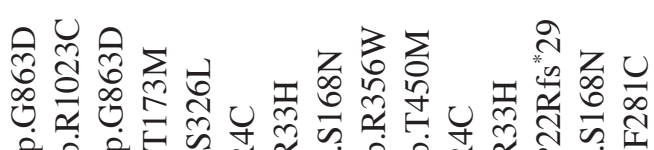

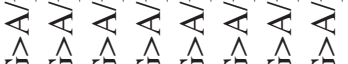

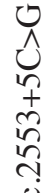

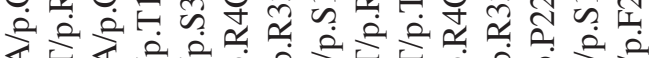

安

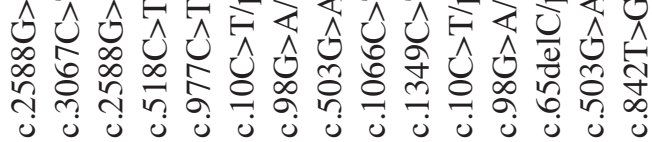

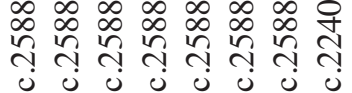

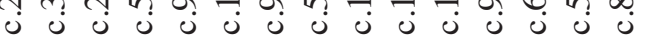

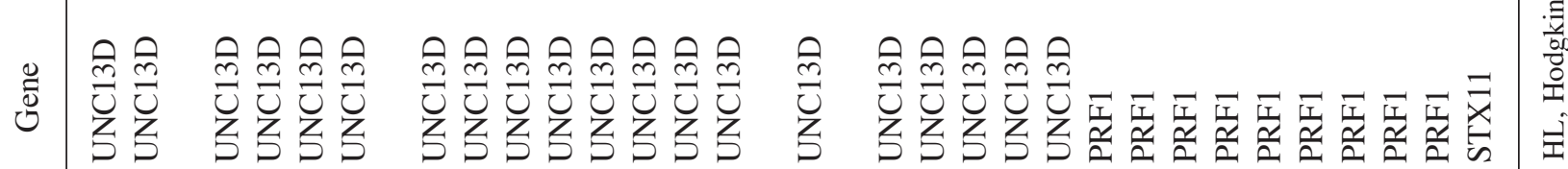

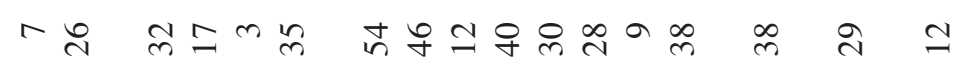

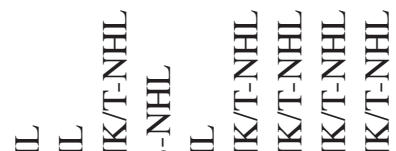

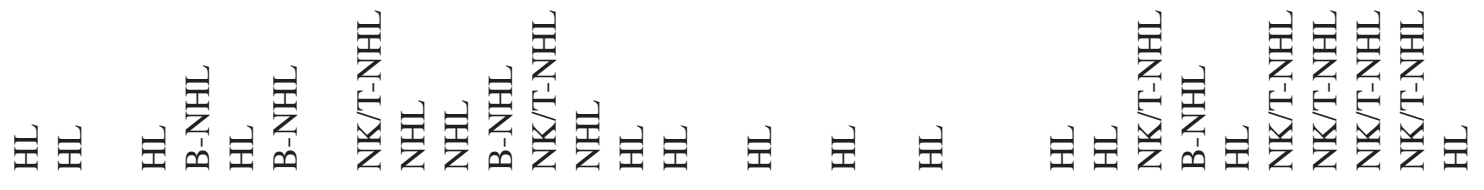

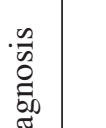

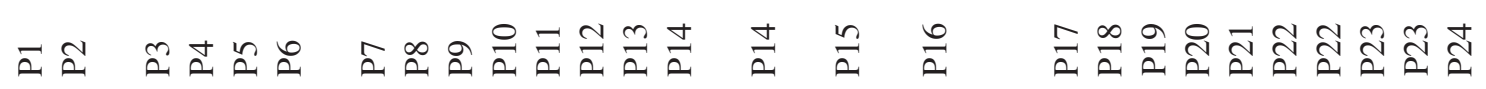

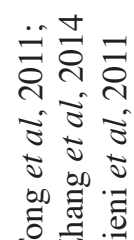

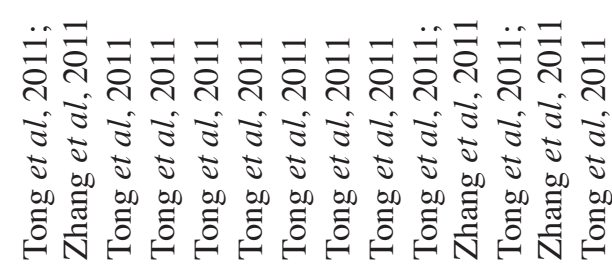

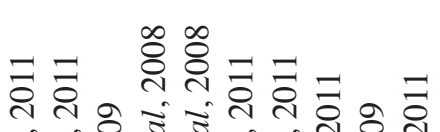

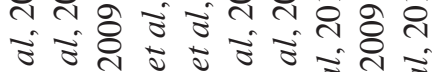

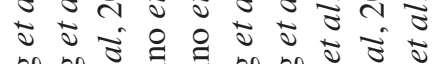

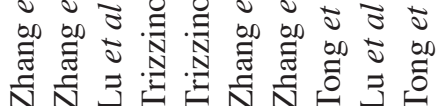


Table III. Allele frequencies of PRF1 c.272T and UNC13D c.2588A among different populations.

Allele frequencies

Populations/samples

PRF1 c.272T

UNC13D c.2588A

1000G-all populations
$1000 \mathrm{G}-\mathrm{CHB}$

$0.0132(66 / 5008)$

$0.0014(7 / 5008)$

1000G-CHS

$0(0 / 206)$

$0.0097(2 / 206)$

1000G-CDX

$0.0048(1 / 210)$

$0.0048(1 / 210)$

1000G-JPT

$0(0 / 186)$

$0.0108(2 / 186)$

1000G-BEB

$0(0 / 208)$

$0(0 / 172)$

$0.0048(1 / 208)$

1000G-FIN

$0.0253(5 / 198)$

$0.0058(1 / 172)$

1000G-GBR

$0.0385(7 / 182)$

$0(0 / 198)$

1000G-TSI

$0.0561(12 / 214)$

Patients in the present study

$0(0 / 180)$

$0(0 / 120)$

$0.0556(10 / 180)$

$0.0095(4 / 420)$

CHB, Han Chinese in Beijing China; CHS, Southern Han Chinese; CDX, Chinese Dai in Xishuangbanna, China; JPT, Japanese in Tokyo Japanese; BEB, Bengali from Bangladesh; FIN, Finnish in Finland; GBR, British in England and Scotland; TSI, Toscani in Italia; UNC13D, unc-13 homolog D; PRF1, perforin; 1000G, 1000 Genomes Project.

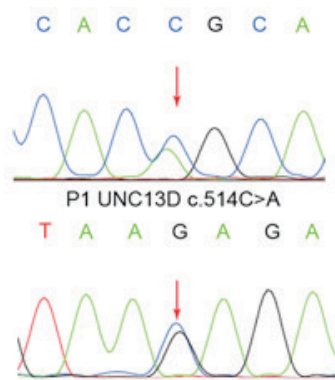

P6 UNC13D c. $2553+5 C>G$
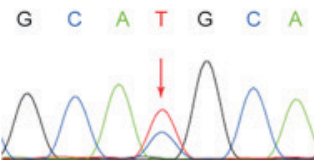

P16 UNC13D c.518C>T
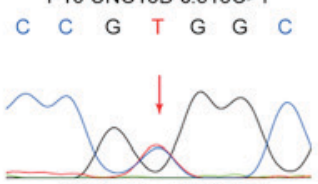

P20 PRF1 c. 1066C>T

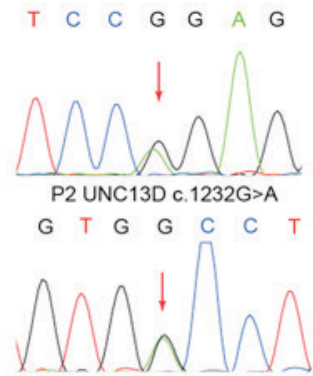

P7 UNC13D c. 2588G $>A$
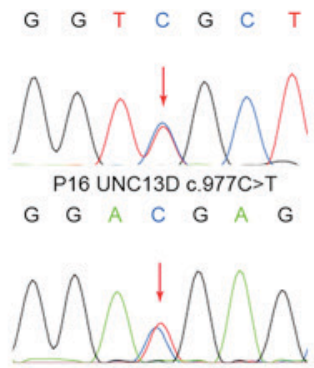

P21 PRF1 c. 1349C>T

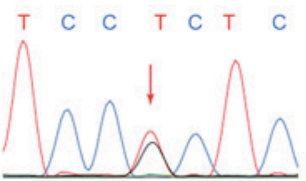

P3 UNC13D c. 1241G $>T$
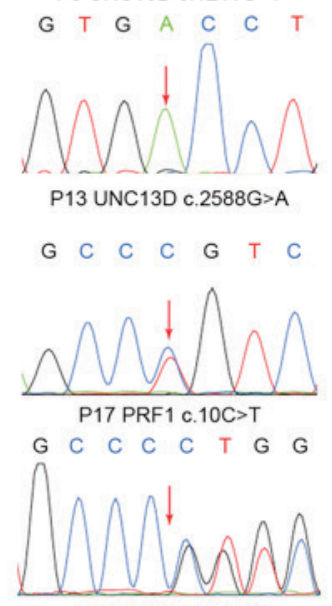

P23 PRF1 c.65delC
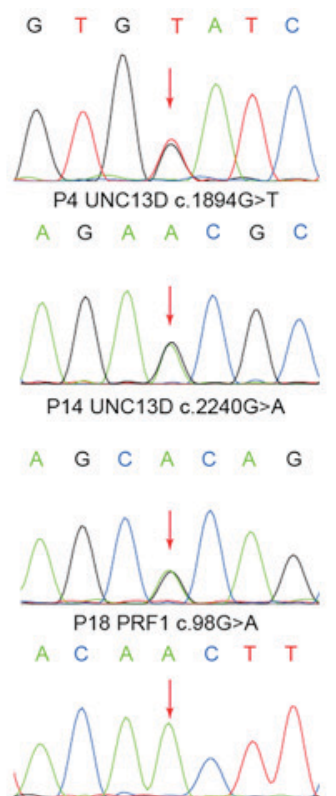

P23 PRF1 c. 503G >A
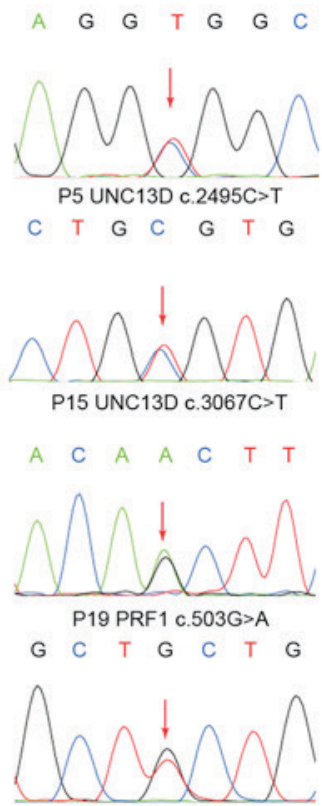

P24 STX11 c.842T>C

Figure 1. Sanger sequencing chromatogram of the genomic polymerase chain reaction product of the 24 patients with lymphoma. Red arrows indicate the mutations detected. UNC13D, unc-13 homolog D; PRF1, perforin; STX11, syntaxin 11; P, patient number.

UNC13D and SH2D1A. Twenty-one patients (25\%) carried PRF1 mutations and the other 2 patients had mutations of UNC13D. PRF1 c. 272C>T/p.A91V mutation was also the most common mutant genotype (11/84).

In the present study 6 genes, which are all involved in cytotoxic function of natural killer cells and cytotoxic T lymphocytes, were identified in 90 Chinese patients with lymphoma. The results demonstrated the association of germline defective mutations and development of lymphoma. The majority of mutations detected in the current study were heterozygous missense mutations, which were consistent with previous reports $(10,11)$. This may explain why these patients developed lymphoma later in life rather than outbreak fatal FHL during infancy. Such monoallelic mutations may contribute to the pathogenesis of the disease, but are not sufficient to initiate the disease phenotype alone. Additional unidentified genetic defects, or possibly even environmental factors, may contribute to the development of lymphoma (10). What was different from reports in Europe was that the most common mutant gene in the present study was UNC13D while PRF1 was less frequently involved, indicating a distinct mutation spectrum in Chinese patients with lymphoma.

Notably, no hot spot region or predominant pathogenic mutation in UNC13D had been previously identified (18). In the current study; however, 9/16 UNC13D mutation 


A Patient
Q70J99_human
H2QDW4_pan troglodytes
G3SJT8_gorilla
H2NUR1_orangutan
F7H074_rhesus
A0A096NSY7_baboon
F7BF78_callithrix Jacchus
HOWJN9_otolemur garnettii
G1QLU3_nomascus leucogenys
A0A0D90XW0_chlorocebus sabaeus
G1LTJ2_ailuropoda melanoleuca
B2RUD2_mouse
Q9R189_rat
HOVLZ9_guinea pig
I3MKW4_squirrel
G1U504_rabbit
E1BFF0_bos taurus
W5PHU7_ovis aries
F1RVY9_sus scrofa
F7AZ96_horse
M3WW4M8_cat
F1PJ91_dog
G3U3P7_elephant
F7EVH3_opossum
M3YQT3_mustela putorius furo
G3WZH3_sarcophilus harrisii
K7EHY3_platypus
F1P5D8_chicken
G1MZPO_meleagris gallopavo
U3J6G6_anas platyrhynchos
U3JE60_ficedula albicollis
H0ZD61_zebra finch
G1KGM2_anolis carolinensis
F7ECT7_western clawed frog
K7FT16_pelodiscus sinensis
H3B2M3_latimeria chalumnae
E6ZG10_dicentrarchus labrax
F6reochromis niloticus

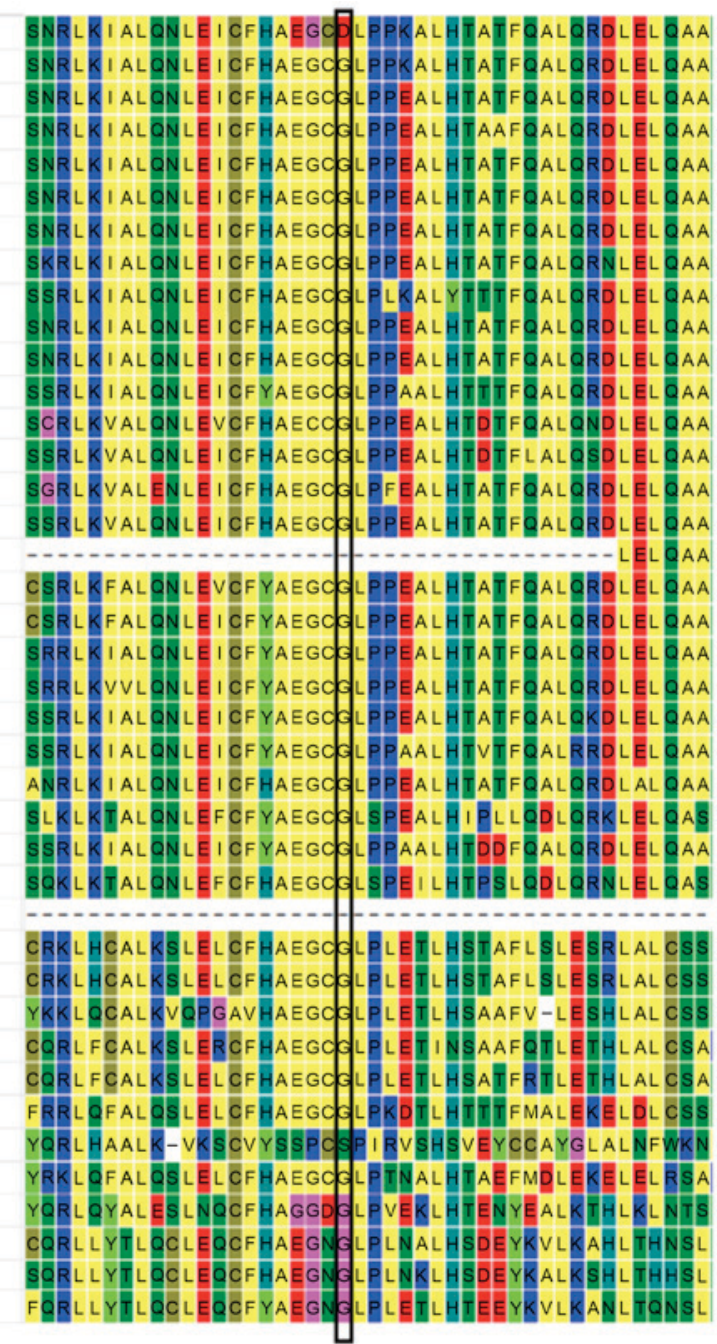
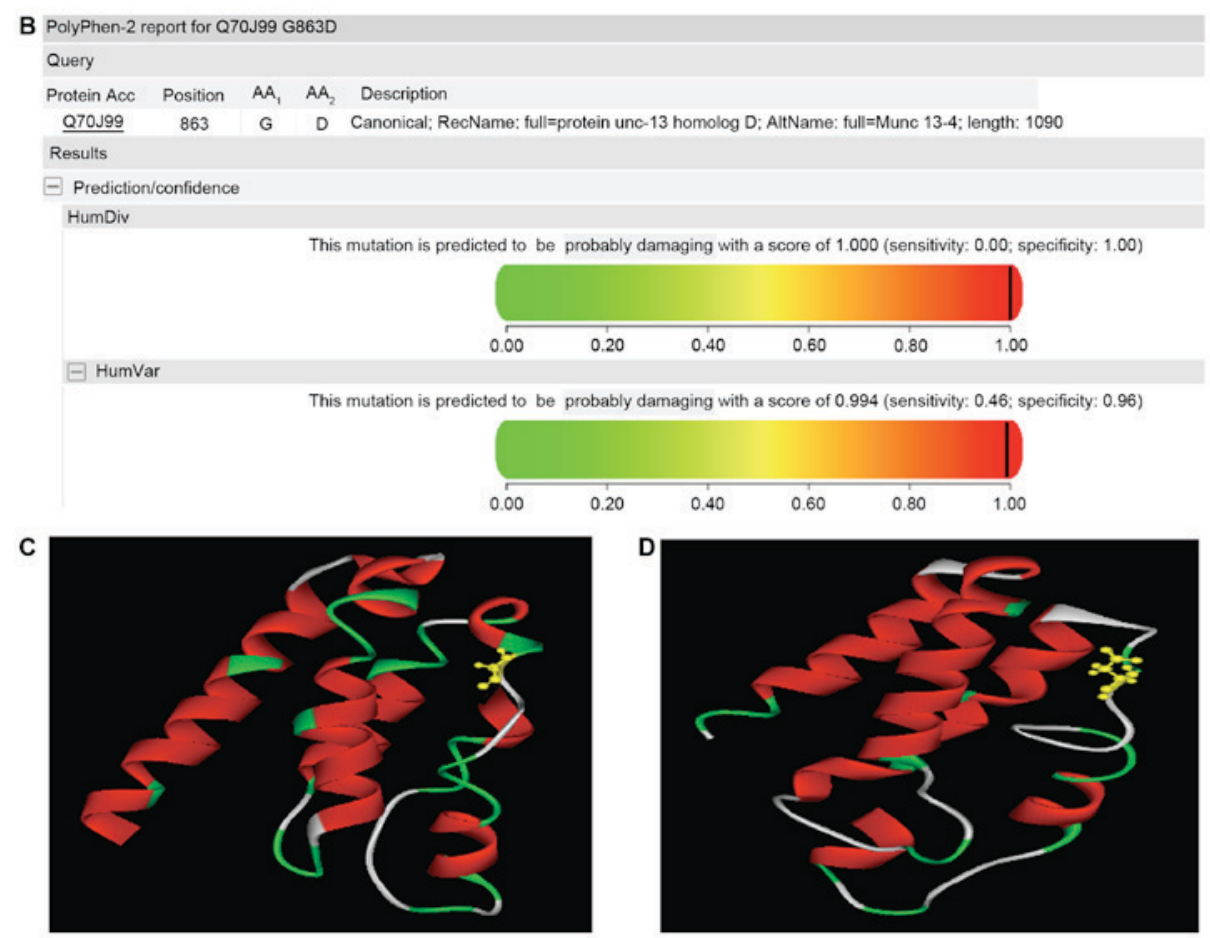

Figure 2. In silico analysis of UNC13D c.2588G $>$ A mutation. (A) Multiple sequence alignment demonstrated that the amino acid at this position was highly conserved in available vertebrate species (Uniprot ID, species). (B) Polymorphism Phenotyping version 2.0 predicted that this mutation is possibly damaging with a score of 0.994. (C) The 3D structure of the wild-type UNC13-4 MHD2. The molecular in yellow is the 863th amino acid of the UNC13-4 protein. (D) 3D structure of the mutant-type UNC13-4 MHD2. The molecular in yellow is the 863th amino acid of the UNC13-4 protein. MHD2, Munc13 homology domain 2; UNC13D, unc-13 homolog D. 
carriers exhibited c.2588G $>$ A/p.G863D mutation, including 1 homozygous and 8 heterozygous. This single amino acid substitution occurred in an evolutionary conserved position and was predicted to be pathogenic using PolyPhen-2, SIFT, and I-TASSER. Furthermore, statistical analysis revealed that this mutation was significantly associated with the risk of developing lymphoma. In addition, none of our patient harbored the PRF1 c.272C $>$ T/p.A91V mutation, which was most frequently reported in European populations $(10,11)$. In the present consecutive cohort of $>500$ patients with diagnosed or suspected FHL, the PRF1 c.272C $>$ T mutation was not identified (data not shown).

Data in the 1000 Genomes Project demonstrated that the allele frequency of PRF1 c272T was significantly higher in European population compared with that in Chinese and Japanese, supporting the concept of a Mediterranean origin of the mutation (11). However, the UNC13D c.2588A allele existed predominantly in Chinese, less in Japanese and Bengali, and was not identified in any other populations listed in this database (Table III). In regards to Korea, where $\mathrm{UNC13D}$ is the predominant causative gene in Korean patients with FHL, c.2588G $>$ A was not reported (19). Collectively, the data obtained from the present study and the databases suggest that UNC13D c.2588G $>A / p . G 863 D$ is a founder mutation of Chinese patients.

In conclusion, the current study provides a relatively comprehensive mutation spectrum of defective cytotoxicity associated genes in Chinese patients with lymphoma. Monoallelic germline mutations were identified to be most frequent in the present cohort, suggesting that partially impaired cytotoxic machinery may represent a predisposing factor for the development of lymphoma. In addition, UNC13D was identified as the predominant causative gene, while PRF1 was less frequently involved. Furthermore, UNC13D c. $2588 \mathrm{G}>\mathrm{A} / \mathrm{p} . \mathrm{G} 863 \mathrm{D}$, which is not reported in other populations, is a founder mutation in Chinese patients.

\section{References}

1. Vesely MD, Kershaw MH, Schreiber RD and Smyth MJ: Natural innate and adaptive immunity to cancer. Annu Rev Immunol 29: 235-271, 2011

2. Tang YM and Xu XJ: Advances in hemophagocytic lymphohistiocytosis: Pathogenesis, early diagnosis/differential diagnosis and treatment. ScientificWorldJournal 11: 697-708, 2011.

3. Stepp SE, Dufourcq-Lagelouse R, Le Deist F, Bhawan S, Certain S, Mathew PA, Henter JI, Bennett M, Fischer A, de Saint Basile G and Kumar V: Perforin gene defects in familial hemophagocytic lymphohistiocytosis. Science 286: 1957-1959, 1999.

4. Feldmann J, Callebaut I, Raposo G, Certain S, Bacq D, Dumont C, Lambert N, Ouachée-Chardin M, Chedeville G, Tamary H, et al: Munc13-4 is essential for cytolytic granules fusion and is mutated in a form of familial hemophagocytic lymphohistiocytosis (FHL3). Cell 115: 461-473, 2003.

5. zur Stadt U, Schmidt S, Kasper B, Beutel K, Diler AS, Henter JI, Kabisch H, Schneppenheim R, Nürnberg P, Janka G and Hennies HC: Linkage of familial hemophagocytic lymphohistiocytosis (FHL) type-4 to chromosome 6q24 and identification of mutations in syntaxin 11. Hum Mol Genet 14: 827-834, 2005.

6. zur Stadt U, Rohr J, Seifert W, Koch F, Grieve S, Pagel J, Strauss J, Kasper B, Nürnberg G, Becker C, et al: Familial hemophagocytic lymphohistiocytosis type 5 (FHL-5) is caused by mutations in Munc18-2 and impaired binding to syntaxin 11. Am J Hum Genet 85: 482-492, 2009.
7. Nichols KE, Harkin DP, Levitz S, Krainer M, Kolquist KA Genovese C, Bernard A, Ferguson M, Zuo L, Snyder E, et al: Inactivating mutations in an $\mathrm{SH} 2$ domain-encoding gene in X-linked lymphoproliferative syndrome. Proc Natl Acad Sci USA 95: 13765-13770, 1998.

8. Rigaud S, Fondanèche MC, Lambert N, Pasquier B, Mateo V, Soulas P, Galicier L, Le Deist F, Rieux-Laucat F, Revy P, et al: XIAP deficiency in humans causes an X-linked lymphoproliferative syndrome. Nature 444: 110-114, 2006.

9. Marsh RA, Madden L, Kitchen BJ, Mody R, McClimon B, Jordan MB, Bleesing JJ, Zhang K and Filipovich AH: XIAP deficiency: A unique primary immunodeficiency best classified as X-linked familial hemophagocytic lymphohistiocytosis and not as X-linked lymphoproliferative disease. Blood 116: 1079-1082, 2010.

10. Clementi R, Locatelli F, Dupré L, Garaventa A, Emmi L, Bregni M, Cefalo G, Moretta A, Danesino C, Comis M, et al: A proportion of patients with lymphoma may harbor mutations of the perforin gene. Blood 105: 4424-4428, 2005.

11. Ciambotti B, Mussolin L, d'Amore ES, Pillon M, Sieni E, Coniglio ML, Ros MD, Cetica V, Aricò M and Rosolen A: Monoallelic mutations of the perforin gene may represent a predisposing factor to childhood anaplastic large cell lymphoma. J Pediatr Hematol Oncol 36: e359-e365, 2014.

12. Tong CR, Liu HX, Xie JJ, Wang F, Cai P, Wang H, Zhu J, Teng W, Zhang X, Yang JF, et al: The study of gene mutations in unknown refractory viral infection and primary hemophagocytic lymphohistiocytosis. Zhonghua Nei Ke Za Zhi 50: 280-283, 2011 (In Chinese).

13. Machaczka M,Klimkowska M, Chiang SC, Meeths M, Müller ML, Gustafsson B, Henter JI and Bryceson YT: Development of classical Hodgkin's lymphoma in an adult with biallelic STXBP2 mutations. Haematologica 98: 760-764, 2013.

14. Sandlund JT, Shurtleff SA, Onciu M, Horwitz E, Leung W, Howard V, Rencher R and Conley ME: Frequent mutations in SH2D1A (XLP) in males presenting with high-grade mature B-cell neoplasms. Pediatr Blood Cancer 60: E85-E87, 2013.

15. World Health Organization (WHO): WHO classification of tumours of haematopoietic and lymphoid tissues. Swerdlow $\mathrm{SH}$, Campo E, Harris NL, Jaffe ES, Pileri SA, Stein H, Thiele J and Vardiman JW (eds). Vol 2. 4th edition. International Agency for Research on cancer (IARC) Press, Lyon, 2008.

16. Ng PC and Henikoff S: SIFT: Predicting amino acid changes that affect protein function. Nucleic Acids Res 31: 3812-3814, 2003.

17. Ramensky V, Bork P and Sunyaev S: Human non-synonymous SNPs: Server and survey. Nucleic Acids Res 30: 3894-3900, 2002.

18. Zur SU, Beutel K, Kolberg S, Schneppenheim R, Kabisch H, Janka G and Hennies HC: Mutation spectrum in children with primary hemophagocytic lymphohistiocytosis: Molecular and functional analyses of PRF1, UNC13D, STX11, and RAB27A. Hum Mutat 27: 62-68, 2006.

19. Yoon HS, Kim HJ, Yoo KH, Sung KW, Koo HH, Kang HJ, Shin HY, Ahn HS, Kim JY, Lim YT, et al: UNC13D is the predominant causative gene with recurrent splicing mutations in Korean patients with familial hemophagocytic lymphohistiocytosis. Haematologica 95: 622-626, 2010.

20. Zhang K, Chandrakasan S, Chapman H, Valencia CA, Husami A, Kissell D, Johnson JA and Filipovich AH: Synergistic defects of different molecules in the cytotoxic pathway lead to clinical familial hemophagocytic lymphohistiocytosis. Blood 124: 1331-1334, 2014.

21. Sieni E, Cetica V, Santoro A, Beutel K, Mastrodicasa E, Meeths M, Ciambotti B, Brugnolo F, zur Stadt U, Pende D, et al: Genotype-phenotype study of familial haemophagocytic lymphohistiocytosis type 3. J Med Genet 48: 343-352, 2011.

22. Zhang K, Jordan MB, Marsh RA, Johnson JA, Kissell D, Meller J, Villanueva J, Risma KA, Wei Q, Klein PS and Filipovich AH: Hypomorphic mutations in PRF1, MUNC13-4, and STXBP2 are associated with adult-onset familial HLH. Blood 118: 5794-5798, 2011.

23. Lu G, Xie ZD, Shen KL, Ye LJ, Wu RH, Liu CY, Jin YK and Yang S: Mutations in the perforin gene in children with hemophagocytic lymphohistiocytosis. Chin Med J (Engl) 122: 2851-2855, 2009.

24. Trizzino A, zur Stadt U, Ueda I, RismaK, Janka G,IshiiE,BeutelK, Sumegi J, Cannella S, Pende D, et al: Genotype-phenotype study of familial haemophagocytic lymphohistiocytosis due to perforin mutations. J Med Genet 45: 15-21, 2008. 